\title{
Editorial briefing
}

\section{HEX goes Open Access}

Welcome to this edition of HEX. Our main news is that, as you might have seen on our website, we have taken the decision to convert Health Expectations to a fully open access journal from 2016. In doing so, $H E X$ will join the Wiley Open Access portfolio (http://www.wileyopenaccess. com) and will benefit from a number of advantages that confers. We made this decision because we all want $H E X$ to continue to publish the very best papers in the field, and we believe that authors will welcome the open access model.

The great advantage of open access is that everyone will be able to read the journal irrespective of specialty, host institution or location. The Article Publication Charge ensures free access to the final published article indefinitely on Wiley Online Library as well as on PubMed Central offering a much wider reach for the research. Authors also get to retain the copyright for their articles.

We want to stress that all Wiley Open Access titles are editorially independent, and this will be the case for $H E X$ too. The editorial team will continue to apply the same rigorous standards of peer review and acceptance criteria, ensuring that the journal remains the high-quality publication we have all come to value enormously.

This issue of $H E X$ should appeal to a broad readership (meeting one of our aims), with a review of the literature reporting parents' experiences of looking after a child with a longterm condition, which highlights the view that parents do not feel their expertise and input is recognized. Watermayer's study reports how the complexities of disclosure of HIV/AIDs with children and adolescents bring about additional challenges for health-care teams. The complementary paper by Penn reports a case study of a recorded consultation in which disclosure of HIV status needed to be made. The analysis illustrates the differing perspectives of the health-care professional, the patient and grandmother, and the counsellor 'acting as a broker between two worlds'. This paper has the potential to be valuable to educators and teachers who want to illustrate the communication skills needed to 'break bad news', as well as to clinicians whose work involves difficult conversations on a regular basis.

Hughes et al. report the development of a tool to assess supportive and palliative care needs, which now needs clinical studies to assess its clinical utility. The importance of this work is that the tool was developed in consultation with 38 consumer and self-help groups - an important source of knowledge and intelligence, which is too often neglected by health-care professionals. Evans' study describes the impact of user involvement in commissioning cancer services - recognizing that such involvement needs to be facilitated and supported. Evans' paper was accepted at the time of the Coalition government - perhaps a further limitation will be how PPI views can be sought and incorporated in commissioning within increasingly constrained resources.

Carolyn A. Chew-Graham on behalf of the Editorial team

\section{Carolyn A. Chew-Graham ${ }^{\star} \dagger$ \\ *Professor of General Practice Research, Research Institute, Primary Care and Health Sciences, Keele University, Staffordshire, UK and †Honorary Professor of Primary Care Mental Health, South Staffordshire and Shropshire Healthcare NHS Foundation Trust, Staffordshire, UK}

